\title{
GMRES ON TRIDIAGONAL BLOCK TOEPLITZ LINEAR SYSTEMS
}

\author{
Reza Doostaki \\ Young Researchers and Elite Club, Kahnooj Branch, Islamic Azad \\ University, Kerman, Iran
}

\begin{abstract}
We study the generalized minimal residual (GMRES) method for solving tridiagonal block Toeplitz linear system $\mathbf{A} x=b$ with $m \times m$ diagonal blocks. For $m=1$, these systems becomes tridiagonal Toeplitz linear systems, and for $m>1, \mathbf{A}$ becomes an $m$-tridiagonal Toeplitz matrix. Our first main goal is to find the exact expressions for the GMRES residuals for $b=\left(B_{1}, 0, \ldots, 0\right)^{T}, b=\left(0, \ldots, 0, B_{N}\right)^{T}$, where $B_{1}$ and $B_{N}$ are $m$-vectors. The upper and lower bounds for the GMRES residuals were established to explain numerical behavior. The upper bounds for the GMRES residuals on tridiagonal block Toeplitz linear systems has been studied previously in [1]. Also, in this paper, we consider the normal tridiagonal block Toeplitz linear systems. The second main goal is to find the lower bounds for the GMRES residuals for these systems.
\end{abstract}

\section{INTRODUCTION}

Iterative methods are used to solve large sparse systems of linear equations $A x=b$. The Generalized Minimal Residual (GMRES) method is such an algorithm and is often used to solve a linear system

$$
A x=b .
$$

The GMRES method starts with an initial approximation $x_{0}$, then computes the initial residual $r_{0}=b-A x_{0}$ and a sequence of approximate solutions $x_{1}, x_{2}, \ldots$, so that the $k$ th residual $r_{k}=b-A x_{k}$ satisfies $([6])$

$$
\left\|r_{k}\right\|_{2}=\min _{x \in x_{0}+\mathcal{K}_{k}\left(A, r_{0}\right)}\|b-A x\|_{2}
$$

2010 Mathematics Subject Classification. 65F10.

Key words and phrases. GMRES, tridiagonal block Toeplitz matrix, linear system. 
where the Krylov subspace $\mathcal{K}_{k}\left(A, r_{0}\right)$ is defined as

$$
\mathcal{K}_{k}\left(A, r_{0}\right)=\operatorname{span}\left\{r_{0}, A r_{0}, \ldots, A^{k-1} r_{0}\right\},
$$

and $\|\cdot\|_{2}$ denotes the $l_{2}$ norm.

Consider the tridiagonal block Toeplitz matrix $\mathbf{A}$ with Toeplitz structure as the form

$$
\mathbf{A}=\left(\begin{array}{cccc}
A_{1} & A_{2} & & \\
A_{3} & \ddots & \ddots & \\
& \ddots & \ddots & A_{2} \\
& & A_{3} & A_{1}
\end{array}\right)_{N \times N}
$$

where the blocks of $\mathbf{A}$ are $m \times m$ diagonal matrices, such as

(1.5) $A_{1}=\left(\begin{array}{ccc}\lambda & & 0 \\ & \ddots & \\ 0 & & \lambda\end{array}\right), A_{2}=\left(\begin{array}{ccc}\mu & & 0 \\ & \ddots & \\ 0 & & \mu\end{array}\right), A_{3}=\left(\begin{array}{ccc}\nu & & 0 \\ & \ddots & \\ 0 & & \nu\end{array}\right)$,

with nonzero parameters $\lambda, \mu$ and $\nu$. Also, let

$$
b=\left(B_{1}, B_{2}, \ldots, B_{N}\right)^{T},
$$

and

$$
B_{l}=\left(b_{(1, l)}, b_{(2, l)}, \ldots, b_{(m, l)}\right)^{T} .
$$

For $m=1$, the matrix $\mathbf{A}$ becomes an $N \times N$ tridiagonal Toeplitz matrix

$$
\mathcal{A}=\left(\begin{array}{cccc}
\lambda & \mu & & \\
\nu & \ddots & \ddots & \\
& \ddots & \ddots & \mu \\
& & \nu & \lambda
\end{array}\right),
$$

and for $m>1$, A becomes an $m$-tridiagonal Toeplitz matrix $\mathcal{A}^{(m)}=\left(a_{i, j}\right)_{i, j=1}^{m N}$ as

$$
\mathcal{A}^{(m)}=\left(\begin{array}{cccccccc}
\lambda & 0 & \ldots & 0 & \mu & 0 & \ldots & 0 \\
0 & \lambda & 0 & \vdots & 0 & \mu & \ddots & \vdots \\
\vdots & 0 & \ddots & 0 & \vdots & \ddots & \ddots & 0 \\
0 & \vdots & \ddots & \lambda & \ddots & \vdots & \ddots & \mu \\
\nu & 0 & \vdots & \ddots & \ddots & \ddots & \vdots & 0 \\
0 & \nu & \ddots & \vdots & 0 & \ddots & 0 & \vdots \\
\vdots & \ddots & \ldots & 0 & \vdots & 0 & \lambda & 0 \\
0 & \ldots & 0 & \nu & 0 & \ldots & 0 & \lambda
\end{array}\right),
$$


where $a_{i, i+m}=\mu, a_{i+m, i}=\nu$ for $1 \leq i \leq m N-m$.

Tridiagonal block Toeplitz linear systems appear in a variety of applications in mathematics, scientific computing, and engineering, such as image processing, numerical differential equations and integral equations, boundary value problems, time series analysis, control theory, etc.

In this paper, we consider the GMRES method on the tridiagonal block Toeplitz linear system $\mathbf{A} x=b$, where $\mathbf{A}$ is defined as (1.4). For case $m=1$, i.e, the tridiagonal Toeplitz linear systems, this problem has been studied previously in $[2-5]$ and the references therein. For $m>1, \mathbf{A}$ becomes an $m$-tridiagonal Toeplitz matrix, and we obtained the upper bounds for the GMRES residuals on linear system $\mathbf{A} x=b$ ([1, Theorem 2.5]). Also, in [1], we showed that the GMRES method on $m N \times m N$ linear system $\mathbf{A} x=b$ computes the exact solution in at most $N$ steps. This paper continues our recent work [1], and the first main goal is to find the exact expressions for the GMRES residuals on the tridiagonal block Toeplitz linear system $\mathbf{A} x=b$, with special right hand side vector

$$
b=\left(B_{1}, 0, \ldots, 0\right)^{T}, \quad b=\left(0, \ldots, 0, B_{N}\right)^{T},
$$

where $B_{1}$ and $B_{N}$ are any $m$-vectors. In fact, we show that the exact expressions for the GMRES residuals for case $m=1$ obtained by [5], are also satisfied for the case $m \geq 1$. The upper and lower bounds for the GMRES residuals were established to explain numerical behavior. The second main goal in this paper, is to find the lower bounds for the GMRES residuals when solving the tridiagonal block Toeplitz linear system $\mathbf{A} x=b$, in case of normal matrix A. We start in analogous way as in [1], and use Chebyshev polynomials of the second kind instead of Chebyshev polynomials of the first kind in [1], to achieve these goals.

Throughout this paper, we denote the $n \times n$ identity matrix by $I_{n}$, and $e_{j}$ is its $j$ th column. The superscript.$^{T}$ denotes transpose of a matrix, and the superscript $*^{*}$ denotes conjugate-transpose of a matrix. For a vector $u$ and a matrix $X, u_{(j)}$ is $j$ th entry of $u, X_{(:, j)}$ is $j$ th column of $X$, and $X_{(:, i: j)}$ consists of intersection of all rows and columns $i$ to $j$, also $\operatorname{diag}(u)$ is the diagonal matrix with $(\operatorname{diag}(u))_{(j, j)}=u_{(j)}$. The Kronecker product of the matrices $A$ and $B$ is given by $A \otimes B=\left[A_{(i, j)} B\right]$. Finally, $\Pi_{k}$ denotes the set of polynomials of degree at most $k$.

\section{BASIC CONCEPTS}

In this section, we start in analogous way as in [1], and major difference between this paper and [1], is in using Chebyshev polynomials of the second kind instead of Chebyshev polynomials of the first kind.

Let the tridiagonal block Toeplitz matrix $\mathbf{A}$ be given as (1.4). The next theorem shows that the matrix $\mathbf{A}$ is diagonalizable when $\mu \neq 0$ and $\nu \neq 0$, and was proven in [1]. 
TheOrem 2.1. The coefficient matrix $\boldsymbol{A}$ defined as (1.4) can be block diagonalized as

$$
\boldsymbol{A}=\tilde{X} \Lambda \tilde{X}^{-1}, \quad \tilde{X}=X_{N} \otimes I_{m}, \quad \Lambda=\operatorname{diag}\left(\Lambda_{1}, \ldots, \Lambda_{N}\right),
$$

where

$$
\begin{gathered}
\Lambda_{j}=\lambda_{j} I_{m}, \quad \lambda_{j}=\lambda-2 \sqrt{\mu \nu} t_{j}, \quad t_{j}=\cos \theta_{j}, \quad \theta_{j}=\frac{j \pi}{N+1}, \\
X_{N}=S Z, S=\operatorname{diag}\left(1, \xi^{-1} \ldots, \xi^{-N+1}\right), \quad \xi=-\frac{\sqrt{\mu \nu}}{\nu} \\
Z_{(:, j)}=\sqrt{\frac{2}{N+1}}\left(\sin j \theta_{1}, \ldots, \sin j \theta_{N}\right)^{T} .
\end{gathered}
$$

It can be verified that $Z^{T} Z=I_{N}$, and $Z^{T}=Z$. Set

$$
\omega=-2 \sqrt{\mu \nu}, \quad \tau=\frac{\lambda}{2 \sqrt{\mu \nu}} .
$$

By (2.2), we have

$$
\lambda_{j}=\omega\left(t_{j}-\tau\right), 1 \leq j \leq N .
$$

The next corollary was proven in [1].

Corollary 2.2. For linear system $\boldsymbol{A} x=b$, where $m N \times m N$ matrix $\boldsymbol{A}$ defined as (1.4), the GMRES method computes the exact solution in at most $N$ steps.

Hence, in this paper, we restrict $k<N$ at all times, where $k$ is the GMRES iteration. From [1, eq. 25], $k$ th GMRES residual $r_{k}$ satisfies

$$
\left\|r_{k}\right\|_{2}=\min _{u_{(1)}=1}\left\|\tilde{X} \operatorname{diag}\left(\tilde{X}^{-1} b\right) \mathcal{V} u\right\|_{2}
$$

where

$$
\mathcal{V}=\left(\begin{array}{c}
\mathcal{V}_{1}^{T} \\
\mathcal{V}_{2}^{T} \\
\vdots \\
\mathcal{V}_{N}^{T}
\end{array}\right)
$$

and $\mathcal{V}_{j}$ are $(k+1) \times m$ Vandermonde matrices as the form

$$
\mathcal{V}_{j}=\left(\begin{array}{cccc}
1 & 1 & \ldots & 1 \\
\lambda_{j} & \lambda_{j} & \ldots & \lambda_{j} \\
\vdots & \vdots & \ddots & \vdots \\
\lambda_{j}^{k} & \lambda_{j}^{k} & \ldots & \lambda_{j}^{k}
\end{array}\right)
$$

Different from [1], we use Chebyshev polynomials of the second kind instead of Chebyshev polynomials of the first kind, to simplify (2.7). 
The Chebyshev polynomials of the second kind of degree $n$ are defined by

$$
U_{m}(t)= \begin{cases}\frac{\sin ((m+1) \arccos t)}{\sin (\arccos t)} & \text { for real } t \text { and }|t| \leq 1, \\ \frac{\left(t+\sqrt{t^{2}-1}\right)^{m+1}-\left(t-\sqrt{t^{2}-1}\right)^{m+1}}{2 \sqrt{t^{2}-1}} & \text { otherwise. }\end{cases}
$$

Also, we define the $n$th Translated Chebyshev polynomial in z of degree $n$ as (2.9) $U_{n}(z ; \omega, \tau) \stackrel{\text { def }}{=} U_{n}\left(\frac{z}{\omega}+\tau\right)=a_{n n} z^{n}+a_{n-1 n} z^{n-1}+\ldots+a_{1 n} z+a_{0 n}$, where $a_{j n}$ are functions of $\omega$ and $\tau$. Let

$$
R_{n}=\left(\begin{array}{cccc}
a_{00} & a_{01} & \cdots & a_{0 n-1} \\
& a_{11} & \cdots & a_{1 n-1} \\
& & \ddots & \vdots \\
& & & a_{n-1 n-1}
\end{array}\right)
$$

where $j$ th column of $R_{n}$ contains the coefficients of $U_{j-1}(z ; \omega, \tau)$.

It can be verified that (see also [1, eq. 39])

$$
\left\|r_{k}\right\|_{2}=\min _{u_{(1)}=1}\left\|\tilde{X} \operatorname{diag}\left(\tilde{X}^{-1} b\right) \Upsilon_{(:, 1: k+1)} R_{k+1}^{-1} u\right\|_{2},
$$

where

$$
\Upsilon=\left(\begin{array}{c}
\Upsilon_{1} \\
\Upsilon_{2} \\
\vdots \\
\Upsilon_{N}
\end{array}\right), \quad \Upsilon_{i}=\left(\begin{array}{cccc}
U_{0}\left(t_{i}\right) & U_{1}\left(t_{i}\right) & \ldots & U_{N-1}\left(t_{i}\right) \\
\vdots & \vdots & \ddots & \vdots \\
U_{0}\left(t_{i}\right) & U_{1}\left(t_{i}\right) & \ldots & U_{N-1}\left(t_{i}\right)
\end{array}\right)_{m \times N}
$$

Let the right-hand side vector $b$ is defined as (1.6). Thus

$$
\tilde{X} \operatorname{diag}\left(\tilde{X}^{-1} b\right) \Upsilon=\sum_{l=1}^{N} \xi^{l-1}\left(S Z \operatorname{diag}\left(Z_{(:, l)}\right) \otimes \operatorname{diag}\left(B_{l}\right)\right) \Upsilon .
$$

We now turn to simplify (2.12). Let $P$ be a permutation matrix as

$$
P=\left(\begin{array}{c}
P_{1} \\
P_{2} \\
\vdots \\
P_{m}
\end{array}\right), \quad P_{i}=\left(\begin{array}{c}
e_{i}^{T} \\
e_{m+i}^{T} \\
\vdots \\
e_{(N-1) m+i}^{T}
\end{array}\right)
$$

hence

$$
P\left(S Z \operatorname{diag}\left(Z_{(:, l)}\right) \otimes \operatorname{diag}\left(B_{l}\right)\right) \Upsilon=\left(\begin{array}{c}
\mathcal{H}_{1} \\
\mathcal{H}_{2} \\
\vdots \\
\mathcal{H}_{m}
\end{array}\right)
$$


where

$$
\left(\mathcal{H}_{i}\right)_{(p, q)}=\frac{2 b_{(i, l)}}{N+1} \sum_{k=1}^{N} \xi^{-p+1} \sin k \theta_{p} \sin l \theta_{k} U_{q-1}\left(t_{k}\right), \quad p, q=1, \ldots, N .
$$

It can be verified that

$$
\mathcal{H}_{i}=b_{(i, l)} S Z \operatorname{diag}\left(Z_{(:, l)}\right) \mathbf{U}
$$

where

$$
\mathbf{U}=\left(\begin{array}{cccc}
U_{0}\left(t_{1}\right) & U_{1}\left(t_{1}\right) & \ldots & U_{N-1}\left(t_{1}\right) \\
U_{0}\left(t_{2}\right) & U_{1}\left(t_{2}\right) & \ldots & U_{N-1}\left(t_{2}\right) \\
\vdots & \vdots & \ddots & \vdots \\
U_{0}\left(t_{N}\right) & U_{1}\left(t_{N}\right) & \ldots & U_{N-1}\left(t_{N}\right)
\end{array}\right)_{N \times N}
$$

Thus, the equations (2.13) and (2.14) yield

$$
\begin{array}{r}
P\left(S Z \operatorname{diag}\left(Z_{(:, l)}\right) \otimes \operatorname{diag}\left(B_{l}\right)\right) \Upsilon_{(:, 1: k+1)} \\
=\left(\begin{array}{c}
b_{(1, l)} S Z \operatorname{diag}\left(Z_{(:, l)}\right) \mathbf{U}_{(:, 1: k+1)} \\
b_{(2, l)} S Z \operatorname{diag}\left(Z_{(:, l)}\right) \mathbf{U}_{(:, 1: k+1)} \\
\vdots \\
b_{(m, l)} S Z \operatorname{diag}\left(Z_{(:, l)}\right) \mathbf{U}_{(:, 1: k+1)}
\end{array}\right),
\end{array}
$$

and from (2.12) and (2.15) we can write

$$
P \tilde{X} \operatorname{diag}\left(\tilde{X}^{-1} b\right) \Upsilon_{(:, 1: k+1)}
$$

$$
=\sum_{l=1}^{N} \xi^{l-1}\left(\begin{array}{c}
b_{(1, l)} S Z \operatorname{diag}\left(Z_{(:, l)}\right) \mathbf{U}_{(:, 1: k+1)} \\
b_{(2, l)} S Z \operatorname{diag}\left(Z_{(:, l)}\right) \mathbf{U}_{(:, 1: k+1)} \\
\vdots \\
b_{(m, l)} S Z \operatorname{diag}\left(Z_{(:, l)}\right) \mathbf{U}_{(:, 1: k+1)}
\end{array}\right)
$$

Moreover, the structure of Chebyshev polynomials of the second kind yields

$$
Z \operatorname{diag}\left(Z_{(:, l)}\right) \mathbf{U}=Z D_{l} Z^{T},
$$

where

$$
D_{l}=\left(\begin{array}{cccc}
\frac{\sin \left(l \theta_{1}\right)}{\sin \left(\theta_{1}\right)} & & & \\
& \frac{\sin \left(l \theta_{2}\right)}{\sin \left(\theta_{2}\right)} & & \\
& & \ddots & \\
& & & \frac{\sin \left(l \theta_{N}\right)}{\sin \left(\theta_{N}\right)}
\end{array}\right) \text {. }
$$

Hence, by combining (2.16) and (2.17), we have

(2.18) $P \tilde{X} \operatorname{diag}\left(\tilde{X}^{-1} b\right) \Upsilon_{(:, 1: k+1)}=\sum_{l=1}^{N} \xi^{l-1}\left(\begin{array}{c}b_{(1, l)} S\left(Z D_{l} Z^{T}\right)_{(:, 1: k+1)} \\ b_{(2, l)} S\left(Z D_{l} Z^{T}\right)_{(:, 1: k+1)} \\ \vdots \\ b_{(m, l)} S\left(Z D_{l} Z^{T}\right)_{(:, 1: k+1)}\end{array}\right)$. 


\section{The EXACT EXPRESSION FOR The GMRES RESIDUALS}

The next theorem gives the exact expression for the GMRES residuals when solving the tridiagonal block Toeplitz linear systems $\mathbf{A} x=b$, where $\mathbf{A}$ and $b$ are defined as (1.4) and (1.9), respectively, and it was proven in [5, Theorem 2.1] only for $m=1$. But in this paper, we show that this theorem is established for general case $m \geq 1$.

Theorem 3.1. Consider the tridiagonal block Toeplitz linear system $\boldsymbol{A} x=b$, where $m N \times m N$ matrix $\boldsymbol{A}$ is defined as (1.4). Then, for $1 \leq k<N$, the $k$ th GMRES residual $r_{k}$ satisfies

$$
\left\|r_{k}\right\|_{2}=\|b\|_{2}\left[\sum_{j=0}^{k}|\xi|^{2 j}\left|U_{j}(\tau)\right|^{2}\right]^{-1 / 2} \quad, \quad \text { for } \quad b=\left(B_{1}, 0, \ldots, 0\right)^{T}
$$

$$
\left\|r_{k}\right\|_{2}=\|b\|_{2}\left[\sum_{j=0}^{k}\left|\xi^{-1}\right|^{2 j}\left|U_{j}(\tau)\right|^{2}\right]^{-1 / 2} \quad, \quad \text { for } \quad b=\left(0, \ldots, 0, B_{N}\right)^{T}
$$

where $B_{1}$ and $B_{N}$ are $m$-vectors, and $U_{j}$ is the $j$ th Chebyshev polynomial of the second kind.

Proof. Let $b=\left(B_{1}, 0, \ldots, 0\right)^{T}$ and $B_{l}=\left(b_{(1, l)}, b_{(2, l)}, \ldots, b_{(m, l)}\right)$. Equation (2.18) yields

$$
P \tilde{X} \operatorname{diag}\left(\tilde{X}^{-1} b\right) \Upsilon_{(:, 1: k+1)} R_{k+1}^{-1}=\left(\begin{array}{c}
b_{(1,1)} S_{k+1} R_{k+1}^{-1} \\
b_{(2,1)} S_{k+1} R_{k+1}^{-1} \\
\vdots \\
b_{(m, 1)} S_{k+1} R_{k+1}^{-1}
\end{array}\right),
$$

where $S_{k+1}=S_{(:, 1, k+1)}$. Then by $(2.11)$, we have

$$
\begin{aligned}
\left\|r_{k}\right\|_{2} & =\min _{u_{(1)}=1}\left\|P \tilde{X} \operatorname{diag}\left(\tilde{X}^{-1} b\right) \Upsilon_{(:, 1: k+1)} R_{k+1}^{-1} u\right\|_{2} \\
& =\left\|B_{1}\right\|_{2} \min _{u_{(1)}=1}\left\|S_{k+1} R_{k+1}^{-1} u\right\|_{2} .
\end{aligned}
$$

From [4, Lemma 3.1]

$$
\min _{u_{(1)}=1}\left\|S_{k+1} R_{k+1}^{-1} u\right\|_{2}=\left[\sum_{j=0}^{k}|\xi|^{2 j}\left|U_{j}(\tau)\right|^{2}\right]^{-1 / 2} .
$$

Thus for $b=\left(B_{1}, 0, \ldots, 0\right)^{T}$, equations (3.3) and (3.4) yield

$$
\left\|r_{k}\right\|_{2}=\left\|B_{1}\right\|_{2}\left[\sum_{j=0}^{k}|\xi|^{2 j}\left|U_{j}(\tau)\right|^{2}\right]^{-1 / 2} .
$$


From [4, Sect. 2.2], any result for the GMRES residuals for $\mathbf{A} x=b$ leads to one for $\mathbf{A}^{T} y=\Pi^{T} b$ after performing the following substitutions

$$
\mu \longleftarrow \nu, \quad \nu \longleftarrow \mu, \quad \xi \longleftarrow \xi^{-1}, \quad b \longleftarrow \Pi^{T} b
$$

where $\Pi=\left(e_{m N}, \ldots, e_{2}, e_{1}\right)$. Hence for $b=\left(0, \ldots, 0, B_{N}\right)^{T}$, we can write

$$
\left\|r_{k}\right\|_{2}=\left\|B_{N}\right\|_{2}\left[\sum_{j=0}^{k}\left|\xi^{-1}\right|^{2 j}\left|U_{j}(\tau)\right|^{2}\right]^{-1 / 2} \text {. }
$$

The next theorem gives the asymptotic speed of $\left\|r_{k}\right\|_{2}$ among all possible $r_{0}$ for the GMRES residuals on solving the tridiagonal block Toeplitz linear systems $\mathbf{A} x=b$, where $\mathbf{A}$ and $b$ are defined as (1.4) and (1.9), respectively.

THEOREM 3.2. Under the conditions of the Theorem 3.1, the asymptotic speed of $\left\|r_{k}\right\|_{2}$ is

$$
\begin{aligned}
\lim _{k \rightarrow \infty} \inf _{N>k}\left[\sup _{r_{0}} \frac{\left\|r_{k}\right\|_{2}}{\left\|r_{0}\right\|_{2}}\right]^{1 / k} & =\lim _{k \rightarrow \infty} \sup _{N>k}\left[\sup _{r_{0}} \frac{\left\|r_{k}\right\|_{2}}{\left\|r_{0}\right\|_{2}}\right]^{1 / k} \\
& =\min \left\{(|\xi| \rho)^{-1}, 1\right\}
\end{aligned}
$$

for $r_{0}=\left(B_{1}, 0, \ldots, 0\right)^{T}$. Also, for $r_{0}=\left(0, \ldots, 0, B_{N}\right)^{T}$, the asymptotic speed is

$$
\begin{aligned}
\lim _{k \rightarrow \infty} \inf _{N>k}\left[\sup _{r_{0}} \frac{\left\|r_{k}\right\|_{2}}{\left\|r_{0}\right\|_{2}}\right]^{1 / k} & =\lim _{k \rightarrow \infty} \sup _{N>k}\left[\sup _{r_{0}} \frac{\left\|r_{k}\right\|_{2}}{\left\|r_{0}\right\|_{2}}\right]^{1 / k} \\
& =\min \left\{\left(|\xi|^{-1} \rho\right)^{-1}, 1\right\}
\end{aligned}
$$

where $\rho=\max \left\{\left|\tau+\sqrt{t^{2}-1}\right|,\left|\tau-\sqrt{t^{2}-1}\right|\right\}$.

Proof. From Theorem 3.1, we have

$$
\frac{\left\|r_{k}\right\|_{2}}{\left\|r_{0}\right\|_{2}}=\left[\sum_{j=0}^{k}|\xi|^{2 j}\left|U_{j}(\tau)\right|^{2}\right]^{-1 / 2}, \quad \text { for } \quad r_{0}=\left(B_{1}, 0, \ldots, 0\right)^{T}
$$

and

$$
\frac{\left\|r_{k}\right\|_{2}}{\left\|r_{0}\right\|_{2}}=\left[\sum_{j=0}^{k}\left|\xi^{-1}\right|^{2 j}\left|U_{j}(\tau)\right|^{2}\right]^{-1 / 2}, \quad \text { for } \quad r_{0}=\left(0, \ldots, 0, B_{N}\right)^{T}
$$

For $m=1$, equations (3.6) and (3.7) was proven for the worst asymptotic speed of $\left\|r_{k}\right\|_{2}$ in [5, Theorem 2.2]. Also, the expressions for $\left\|r_{k}\right\|_{2} /\left\|r_{0}\right\|_{2}$ in equations (3.8) and (3.9), are equal to the expressions for $\left\|r_{k}\right\|_{2}$ in [5, Theorem 2.2]. Thus, equations (3.6) and (3.7) are established for $\left\|r_{k}\right\|_{2} /\left\|r_{0}\right\|_{2}$, and this completes the proof. 


\section{GMRES ON NORMAL TRIDIAGONAL BLOCK TOEPLitz LinEAR SystemS}

In this section, let $\mathbf{A}$ be normal. In fact $|\mu|=|\nu|$ is a sufficient and necessary condition for $A$ to be normal. The upper bounds for the GMRES residuals on tridiagonal block Toeplitz linear systems has been studied previously in [1]. In the next Theorem, we obtain the lower bounds for the GMRES residuals on linear system $\mathbf{A} x=b$.

TheOrem 4.1. Let $\boldsymbol{A}$ be the tridiagonal block Toeplitz matrix as in (1.4). Also, let $\boldsymbol{A}$ be normal, then the kth GMRES residual $r_{k}$ satisfies for $1 \leq k<N$

$$
\left\|r_{k}\right\|_{2} \geq \min _{i}\left|D_{(i)}\right|\left[\sum_{j=0}^{k}\left|U_{j}(\tau)\right|^{2}\right]^{-1 / 2},
$$

where $D=\left(Z S^{-1} \otimes I_{m}\right) b$.

Proof. To prove the theorem, we first let $r_{k}$ and $\widetilde{r_{k}}$ be the $k$ th GMRES residuals for normal tridiagonal block Toeplitz linear systems $\mathbf{A} x=b$ and $\mathbf{A} x=\widetilde{b}$, respectively. Because $\mathbf{A}$ is normal, then $|\xi|=1$ and

$$
\left(X_{N} \otimes I_{m}\right)\left(X_{N} \otimes I_{m}\right)^{*}=\left(X_{N} \otimes I_{m}\right)^{*}\left(X_{N} \otimes I_{m}\right)=I_{m N},
$$

i.e., $\left(X_{N} \otimes I_{m}\right)$ is unitary matrix. Hence $\left\|\left(X_{N} \otimes I_{m}\right)\right\|_{2}=1$. Thus equation (2.7) yields

$$
\begin{aligned}
\left\|\widetilde{r_{k}}\right\|_{2}= & \min _{u_{(1)}=1}\left\|\operatorname{diag}\left(\left(X_{N} \otimes I_{m}\right)^{-1} \widetilde{b}\right) \mathcal{V} u\right\|_{2}, \\
= & \min _{u_{(1)}=1} \| \operatorname{diag}\left(\left(X_{N} \otimes I_{m}\right)^{-1} \widetilde{b}\right) \\
& {\left[\operatorname{diag}\left(\left(X_{N} \otimes I_{m}\right)^{-1} b\right)\right]^{-1}\left[\operatorname{diag}\left(\left(X_{N} \otimes I_{m}\right)^{-1} b\right)\right] \mathcal{V} u \|_{2} . }
\end{aligned}
$$

Thus, we can write

(4.4) $\left\|\widetilde{r_{k}}\right\|_{2} \leq\left\|\operatorname{diag}\left(\left(X_{N} \otimes I_{m}\right)^{-1} \widetilde{b}\right)\right\|_{2}\left\|\left[\operatorname{diag}\left(\left(X_{N} \otimes I_{m}\right)^{-1} b\right)\right]^{-1}\right\|_{2}\left\|r_{k}\right\|_{2}$.

Set $\widetilde{b}=e_{1}$, then by Theorem 3.1 , we have

$$
\left\|\widetilde{r_{k}}\right\|_{2}=\left[\sum_{j=0}^{k}\left|U_{j}(\tau)\right|^{2}\right]^{-1 / 2} .
$$

Also, it can be verified that

$$
\begin{aligned}
\left\|\operatorname{diag}\left(\left(X_{N} \otimes I_{m}\right)^{-1} \widetilde{b}\right)\right\|_{2} & =\sqrt{\frac{2}{N+1}} \max \left(\sin \theta_{i}\right), \quad i=1, \ldots, N, \\
& \leq 1 .
\end{aligned}
$$


Thus, equations (4.4), (4.5) and (4.6) yield

$$
\left\|r_{k}\right\|_{2} \geq \sigma_{\min }\left(\operatorname{diag}\left(\left(X_{N} \otimes I_{m}\right)^{-1} b\right)\right)\left[\sum_{j=0}^{k}\left|U_{j}(\tau)\right|^{2}\right]^{-1 / 2}
$$

and this complete the proof.

4.1. Numerical Examples. We give numerical examples which show the lower bounds by Theorem 4.1. All the tests are performed by MATLAB 7.8.

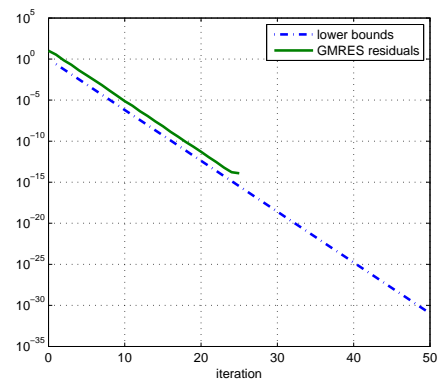

(a) $N=50, m=2, \lambda=2, \mu=0.51$, $\nu=-0.51$

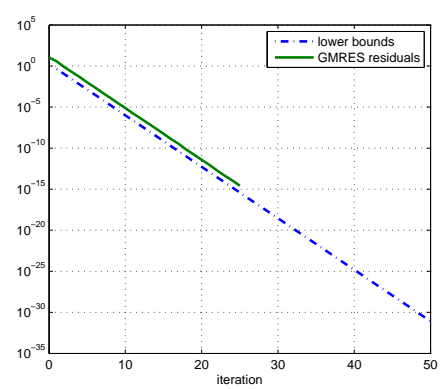

(c) $N=50, m=2, \lambda=i, \mu=0.25 i$, $\nu=-0.25 i$

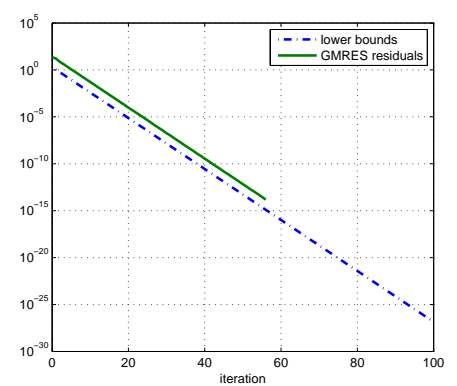

(b) $N=100, m=5, \lambda=4, \mu=3$, $\nu=-3$

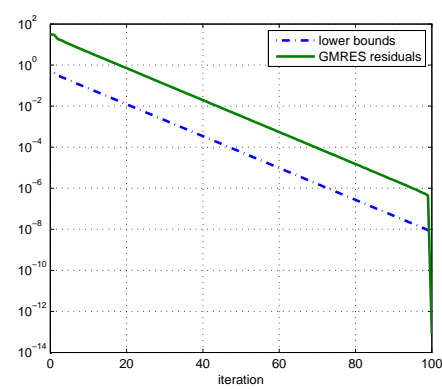

(d) $N=100, m=10, \lambda=1, \mu=2 i$, $\nu=-2$

FigURE 1. GMRES residuals, and their lower bounds by Theorem 4.1 for Example 4.2. 
Example 4.2. Consider the tridiagonal block Toeplitz linear system $\mathrm{A}=\mathrm{b}$, where

$$
\mathbf{A}=\left(\begin{array}{cccc}
A_{1} & A_{2} & & \\
A_{3} & \ddots & \ddots & \\
& \ddots & \ddots & A_{2} \\
& & A_{3} & A_{1}
\end{array}\right)_{n \times n} \quad, \quad A_{1}=\lambda I_{m}, \quad A_{2}=\mu I_{m}, \quad A 3=\nu I_{m}
$$

and each of $b$ 's entries being uniformly random in $[-1,1]$. Figure 1 plots the GMRES residuals on $\mathbf{A} x=b$ and their lower bounds (dashed lines) by Theorem 4.1 for given parameters $\lambda, \mu$ and $\nu$. Also $i$ is the imaginary unit. As mentioned in [1, Cor. 2.3], the GMRES method on $m N \times m N$ linear system $\mathbf{A} x=b$, computes the exaxt solution in at most $N$ steps. For this reason, in each of the subfigures, we set maximum of the iterations equal to $N$.

\section{REFERENCES}

[1] R. Doostaki, Convergence rate of GMRES on tridiagonal block Toeplitz linear systems, Linear Multilinear Algebra 64 (2016), 2533-2544.

[2] R. Doostaki and H. S. Goughery, The upper and lower bounds for generalized minimal residual method on a tridiagonal Toeplitz linear system, Int. J. Comput. Math. 93 (2016), 567-577.

[3] R. C. Li, Convergence of CG and GMRES on a tridiagonal Toeplitz linear system, BIT 47 (2007), 577-599.

[4] R. C. Li and W. Zhang, The rate of convergence of GMRES on a tridiagonal Toeplitz linear system, Numer. Math. 112 (2009), 267-293.

[5] R. C. Li and W. Zhang, The rate of convergence of GMRES on a tridiagonal Toeplitz linear system II, Linear Algebra Appl. 431 (2009), 2425-2436.

[6] Y. Saad and M. Schultz, GMRES: a generalized minimal residual algorithm for solving nonsymmetric linear systems, SIAM J. Sci. Statist. Comput. 7 (1986), 856-869.

R. Doostaki

Young Researchers and Elite Club

Kahnooj Branch, Islamic Azad University

Kerman

Iran

E-mail: rdoostaki@yahoo.com

Received: 14.3.2017.

Revised: 17.11.2017. 